\title{
Deformation of Aluminum Bicrystals with a Composite Crystal Oriented for a Schmid Factor of 0.5 in the Low Strain Range less than $10^{-3}$
}

\author{
By Hiroshi Fujita*, Katsuyuki Toyoda* and Yutaka Kanetsuki**
}

\begin{abstract}
Tilt- and twist-type bicrystals with various misfit angles have been extended along a direction perpendicular to the boundary up to $10^{-3}$ strain. One composite crystal (crystal A) in the specimens is always oriented for a Schmid factor of $\mathbf{0 . 5}$, and another one (crystal B) is systematically rotated against crystal $\mathbf{A}$.

In these specimens, deformation always starts in crystal $\mathbf{A}$, and the interaction between the two crystals considerably occurs even in the low strain range less than $10^{-3}$. In the interaction, the continuity of slip between these two crystals plays an important role. Namely, suitable slips for relieving shear stress associated with the active slip in the opposite crystal are activated near the boundary. Considerable work hardening results from the interactions among these different slip systems even when the strain is less than $10^{-3}$.

The boundary strength increases with increasing misfit angle up to about $20^{\circ}$ in both types of specimens, and then it becomes constant in the range of higher misfit-angles.
\end{abstract}

(Received July 4, 1974)

\section{Introduction}

Mechanical properties of polycrystalline metals are practically important. The strength of metals, however, has been studied mainly using single crystals and consequently the deformation mechanism of polycrystals is not sufficiently clarified yet.

In order to discuss the strength of polycrystalline metals, the following factors must be taken into account in addition to behaviors of single crystals: (1) Orientation factor of polycrystals, (2) the strength of boundaries, (3) interactions among neighboring grains, and (4) heterogeneity of grain sizes.

On the other hand, there are two types of current experiments about the strength of polycrystalline metals. One is symborized by the Hall-Petch relation ${ }^{(1)(2)}$ in which the strength is treated as a function of average grain size. The other has been carried out using bicrystals $^{(3) \sim(6)}$, by which fundamental problems

* Department of Metallurgical Engineering and Materials Science, Faculty of Engineering, Osaka University, Suita 565; Japan.

** Graduate School, Osaka University, Suita. Present address: Asada Fundamental Research Lab., Kobe Steel Co., Ltd., Kobe 657, Japan.

Trans. JIM of polycrystals, such as factors $(1) \sim(3)$, are effectively examined.

The effect of boundary on the strength of bicrystals is due to (a) the interaction between two crystals through the boundary, which is related to the geometrical relationship between two crystals, and (b) the strength of boundary itself ${ }^{(7)}$. Bicrystals with a symmetrical boundary frequently used for studying these two parameters, because the structure of boundaries and the geometrical relationship become simple compared with those of the asymmetrical ones. In such symmetrical bicrystals, however, the active slip systems in two crystals are changed according to the misfit angle of the boundary, and thus not only the mutual interaction between two crystals but also the interaction between active dislocations and boundary are changed. Therefore, the mechanical properties of symmetrical bicrystals become rather complex with increasing misfit angle of the boundary.

This is a reason why, in the present experiment, one of the composite crystals is always oriented for a Schmid factor of 0.5 , and another one is systematically rotated against the fixed crystal. In this case, it is expected that the deformation always starts in the former crystal and transmits into the latter through the

1975 Vol. 16 
boundary. Therefore, not only the strength of boundary but also the interaction between two crystals will be accurately clarified by using this type of bicrystals.

\section{Specimens and Experimental Procedures}

Seed-crystals of $6 \mathrm{~mm} \phi$ were previously grown by the Bridgman method so that their axial directions always were parallel to the [112] axis. Various bicrystals were grown using a

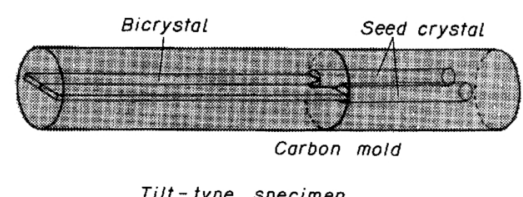

Tilt-type specimen

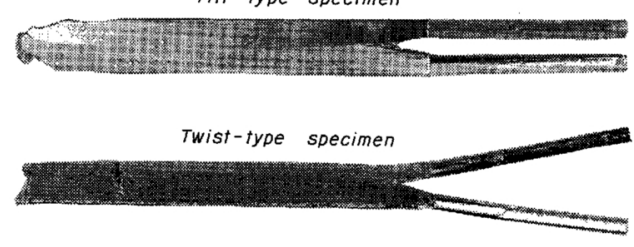

$2 \underline{\mathrm{cm}}$

Fig. 1 Bicrystals grown by using a couple of seed crystals.
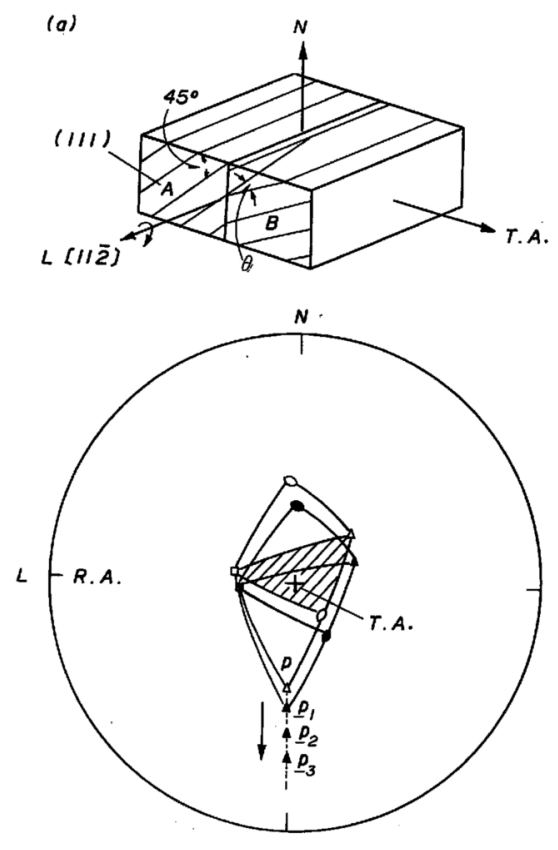

couple of seed-crystals, as shown in Fig. 1. Here one seed crystal was fixed in a soft carbon mold so that one composite crystal of the specimen was always oriented for a Schmid factor of 0.5. Another seed crystal was systematically rotated against the fixed seed crystal $^{(8)}$ around the [112] axis for tilt-type boundaries, as shown in Fig. 2(a), and around the [110] axis for twist-type ones, as shown in Fig. 2(b). Bicrystals of $1.5 \times 20 \times 200 \mathrm{~mm}^{3}$ in size were grown from these couples of seeds by the Bridgman method in a vacuum of $10^{-6} \mathrm{mmHg}$, as shown in Fig. 1. Here, a crystal oriented for Schmid factor of 0.5 is named "crystal A" and the other "crystal B". The misfit angles of bicrystals are shown in Table 1, which were ascertained by X-rays.

The specimens of $1.5 \times 20 \times 20 \mathrm{~mm}^{3}$ were cut from these mother bicrystals with an acid

Table 1 Misfit angles of both types of specimens.

\begin{tabular}{lrrrrrrrrrr}
\hline \hline Type & \multicolumn{1}{c}{ Misfit angle (degree) } \\
\hline Tilt & 7 & 8 & 10 & 12 & 15 & 19 & 24 & 37 & 43 & 97 \\
Twist & 5 & 10 & 17 & 20 & 25 & & & & & \\
\hline \hline
\end{tabular}
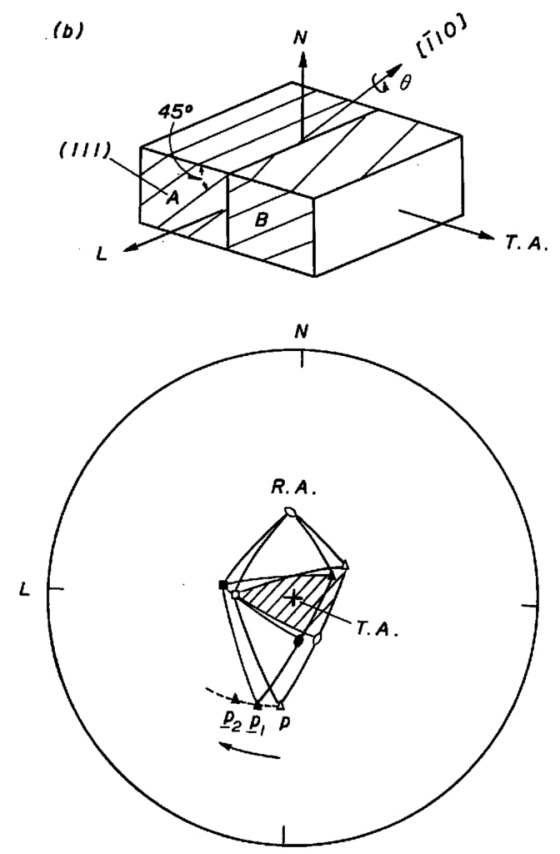

Fig. 2 Geometrical relationships in tilt- and twist-type bicrystals. T.A.: tensile axis, R.A.: rotation axis, $\theta$ : misfit angle, N: specimen surface normal. (a) Tilt-type bicrystals (b) Twisttype bicrystals. 
cutter, and set on a four-point bending device after electropolishing so that their stress axes are perpendicular to the boundary, as shown in Fig. 2.

With a four-point bending device ${ }^{(9)}$ crystals $\mathbf{A}$ and $\mathbf{B}$ were extended along a tensile direction perpendicular to the boundary on the top surface of the specimen. Since the amount of strain is the same at both the top (tension side) and the bottom (compression side) surfaces of the specimen, the strain was measured on the bottom surface with a wire strain-gauge, KYOWA K$3 \mathrm{AI}-23$ of $3 \mathrm{~mm}$ gauge length, stuck across the boundary. The wire strain-gauge was also stuck on each of individual crystals $\mathbf{A}$ and $\mathbf{B}$ in order to measure the strain in each crystal. On the top surface, observation of slip bands was made continuously by optical microscopy during deformation. Applied load was measured by the strain of a coil spring with a dial gauge.

\section{Experimental Results}

\section{Tilt-type specimens}

Figures $3 \sim 6$ show the stress-strain curves of tilt-type specimens whose misfit angles $(\theta)$ are $7,19,24$ and $97^{\circ}$, respectively. In these curves, $\sigma_{\mathrm{A}}\left(\sigma_{\mathrm{B}}\right)$ shows the yield stress of crystal $\mathbf{A}(\mathbf{B})$, and $\sigma_{\mathrm{A}-\mathrm{B}}\left(\sigma_{\mathrm{B}-\mathrm{A}}\right)$ shows a stress at which deformation is transmitted remarkably from crystal A to B (or vice versa). Furthermore, each series of micrographs in Figs. $3 \sim 6$ shows the development of slip bands with an increase
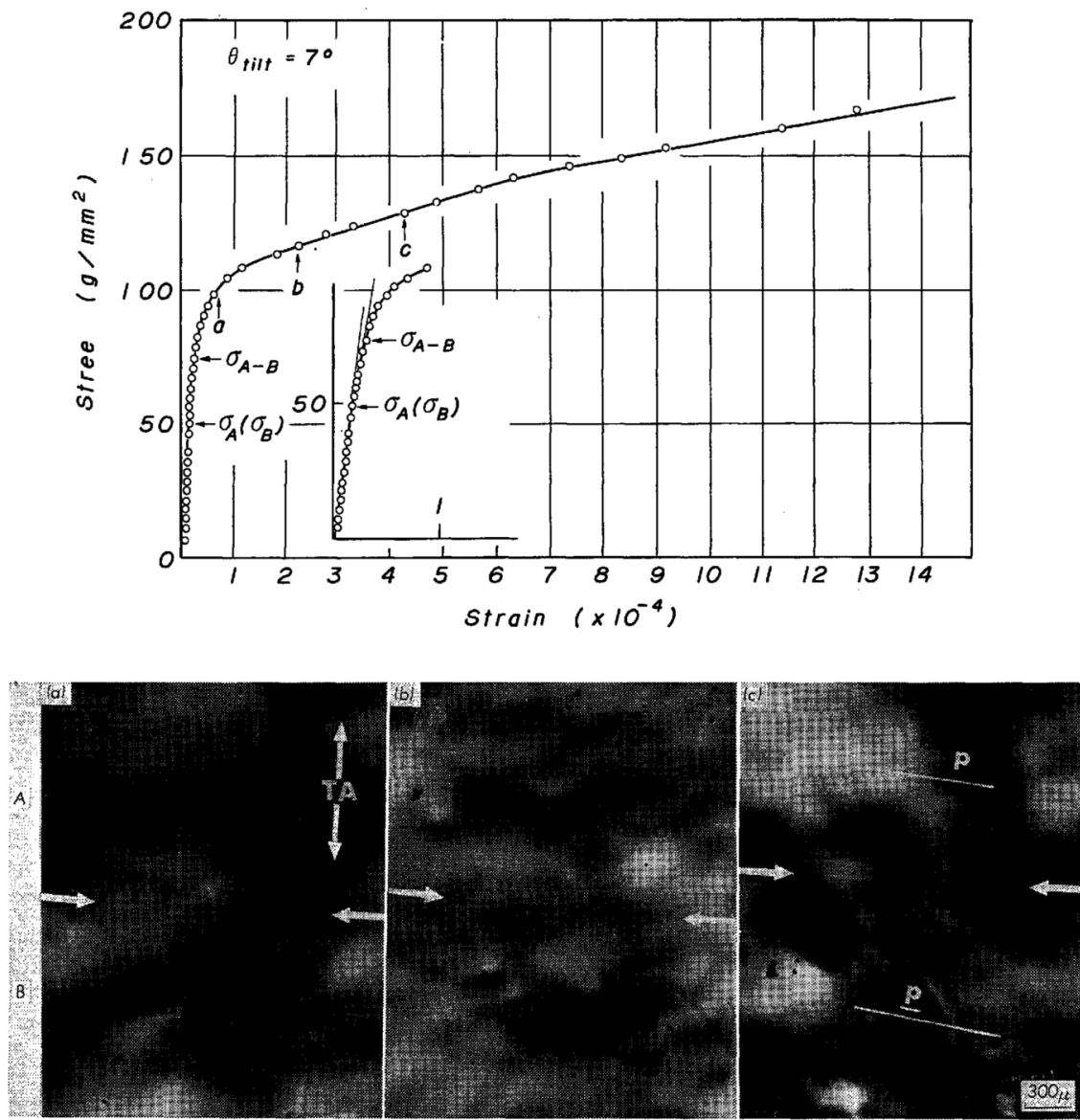

Fig. 3 Deformation of a tilt-type bicrystal with $\theta=7^{\circ}$. Micrographs (a) $\sim$ (c) show the slip bands corresponding to strain $\mathrm{a}, \mathrm{b}$ and $\mathrm{c}$ in the stress-strain curve, respectively. A pair of white arrows indicates the position of boundary, and the tensile stress is applied along a direction (T.A.) perpendicular to the boundary. 
of the stress, and micrographs (a), (b) and (c) were taken at strains $a, b$ and $c$ in the stressstrain curves, respectively. In these micrographs, a boundary is indicated by a pair of arrows, and slip notations ( $p, c o n j, c r i t$ and cross) and ( $\underline{p}$, conj, crit and cross) show the primary, conjugate, critical and cross slip in crystal $\mathbf{A}$ and corresponding ones in crystal $\mathbf{B}$, respectively.

Furthermore, the slip system at each strain is determined from the appearance of slip bands in both crystals. These slip bands are difficult to observe at the early stage of deformation in general, and thus they are determined from those at high strains in the strain range in which the stress-strain relation is monotonously changed, as shown in Figs. $3 \sim 6$.

Determination of $\sigma_{\mathrm{A}}, \sigma_{\mathrm{B}}, \sigma_{\mathrm{A}-\mathrm{B}}$ and $\sigma_{\mathrm{B}-\mathrm{A}}$ has been made from slope changes in the stressstrain curves, as shown in an inserted curve in Fig. 3. The abscissa in Fig. $3 \sim 6$ is reduced in scale in order to illustrate a wide range of strain. In Figs. $3 \sim 6$, it is noted that $\sigma_{\mathrm{A}}$ is always constant, i.e., about $50 \mathrm{~g} / \mathrm{mm}^{2}$ in tensile stress. On the other hand, $\sigma_{\mathrm{B}}$ increases with increasing $\theta$ up to about $15^{\circ}$, and then decreases.

In Fig. $3\left(\theta=7^{\circ}\right)$, the primary slip in crystals $\mathbf{A}$ and $\mathbf{B}$ is $p$ and $\underline{p}$, respectively. These primary slip bands are observed only in crystal $\mathbf{A}$ at strain $b$, and in both crystals $\mathbf{A}$ and $\mathbf{B}$ at strain $c$. In the stress-strain curve, a small slope change also appears at a stress $\sigma_{\mathrm{A}-\mathrm{B}}$, though the main slip systems $\underline{p}$ and $\underline{p}$ in both crystals do not change. And thus, it is considered that $\sigma_{\mathrm{A}-\mathrm{B}}$ corresponds to a stress at which the dislocations of slip $p$ in crystal $\mathbf{A}$ transmit to the corresponding slip planes of slip $\underline{p}$ in crystal $\mathbf{B}$ through the boundary. In the stress range higher than $\sigma_{\mathrm{A}-\mathrm{B}}$, the flow stress increases monotonously with increasing amount of strain up to the order of
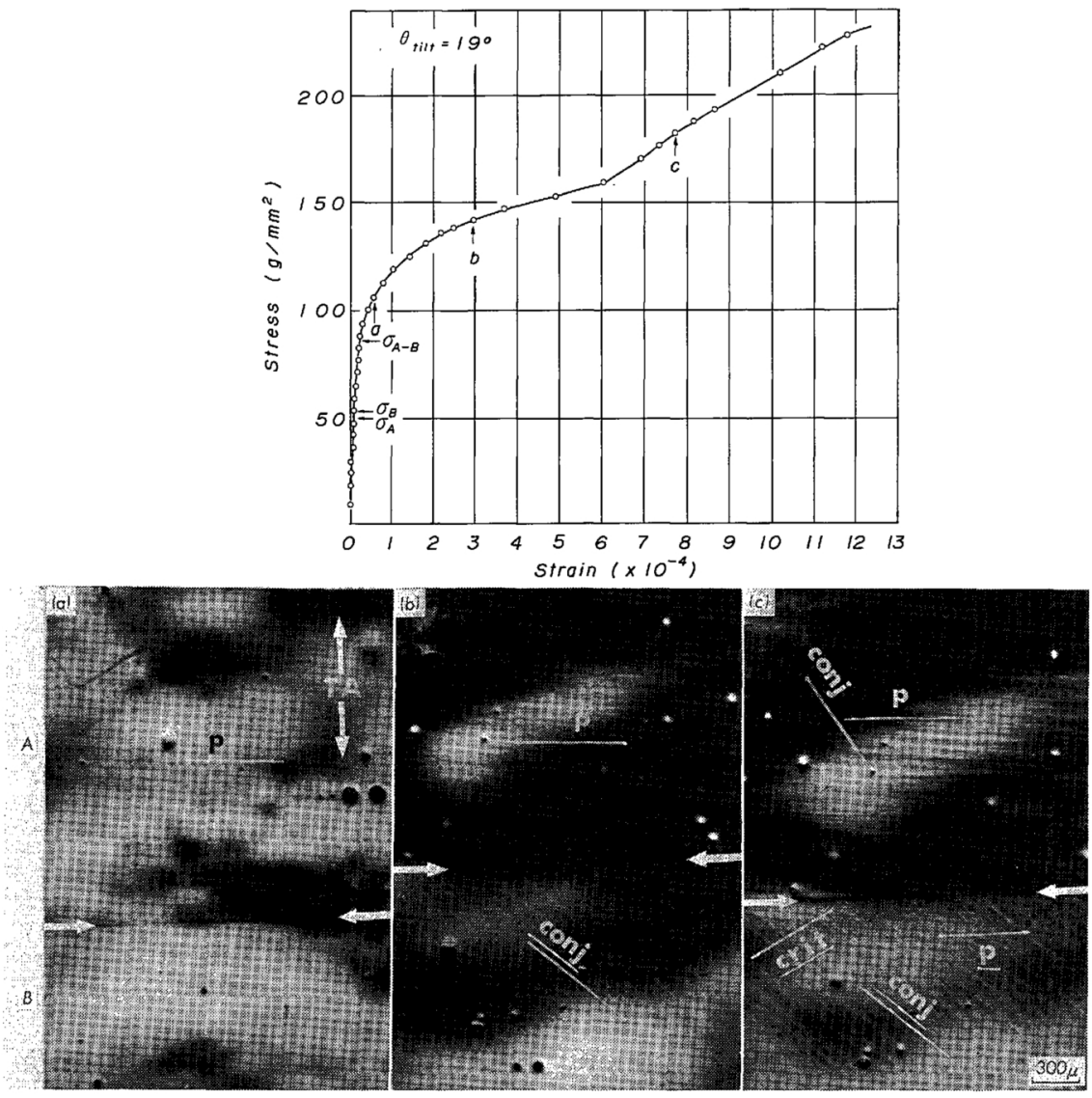

Fig. 4 Deformation of a tilt-type bicrystal with $\theta=19^{\circ}$. 
$10^{-3}$ strain which corresponds to the macroscopic yield point.

When the misfit angle $\theta$ increases to $10^{\circ}$, the situation is changed although the primary slips in both crystals are the same as in a case of $\theta=$ $7^{\circ}$. Namely, the stress level monotonously increases with increasing strain up to $8 \sim 9 \times$ $10^{-4}$, but it abruptly increases when the strain reaches about $1.2 \times 10^{-3}$. In the corresponding micrographs, the primary slip $(p)$ and the corresponding primary slip $(\underline{p})$ appear successively in crystals $\mathbf{A}$ and $\mathbf{B}$, respectively. In this case, the amount of slip $p$ in crystal $\mathbf{A}$ is small in regions near the boundary, but slip $\underline{p}$ in crystal B appears prominently near the boundary. At a strain, of $1.2 \times 10^{-3}$, the conjugate slip (conj) appears near the boundary in crystal $\mathbf{A}$ and the corresponding conjugate slip ( $\underline{\text { conj })}$ ) all over crystal $\mathbf{B}$ in addition to slips $p$ and $\underline{p}$, respectively. And it is thus considered that the

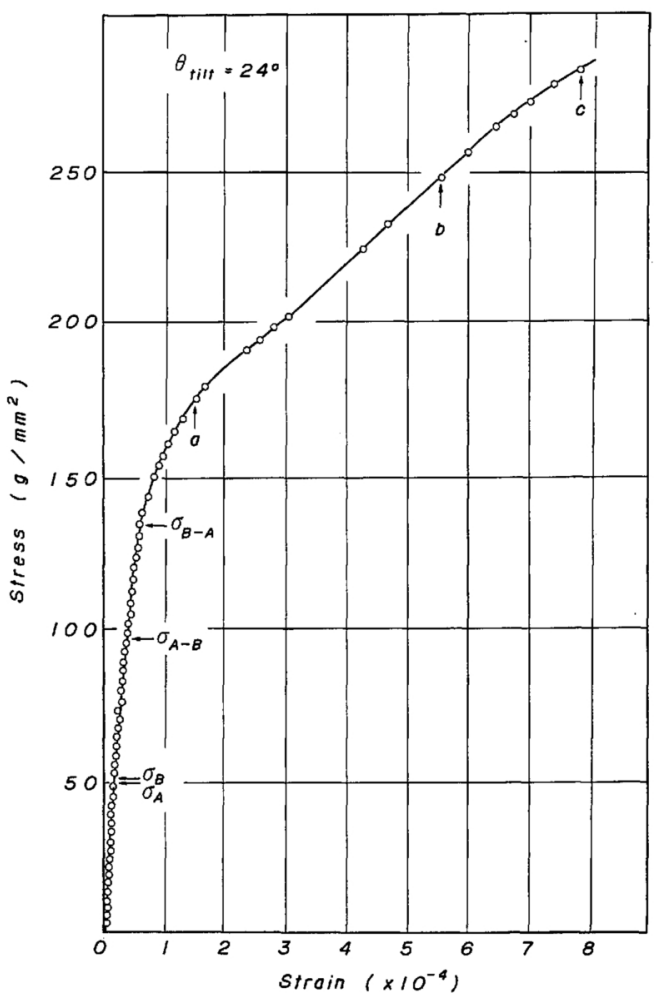

abrupt increase of stress in this case results from the interaction between two slip systems $p$ and $c o n j$ and between $\underline{p}$ and $c o n j$.

The interaction between the two crystals becomes more prominent when $\theta$ increases to $19^{\circ}$, as seen in Fig. 4. Namely, the primary slip $p$ appears at first in crystal $\mathbf{A}$ as seen in micrograph (a), and then slips conj and conj are formed as seen in micrograph (b). After that, $\underline{p}$ and crit are formed in narrow regions near the boundary in crystal $\mathbf{B}$, as seen in micrograph (c). In this case, it should be noted that the amount of slip $p$ is rather small in regions near the boundary. The slip bands of $\underline{p}$ and crit are short in length and show an appearance of wavy slip by combination with slip conj. Since the abrupt increase of flow stress in Fig. 4 corresponds to the formation of these secondary slip bands, it is also considered that the rapid hardening is due to the interac-

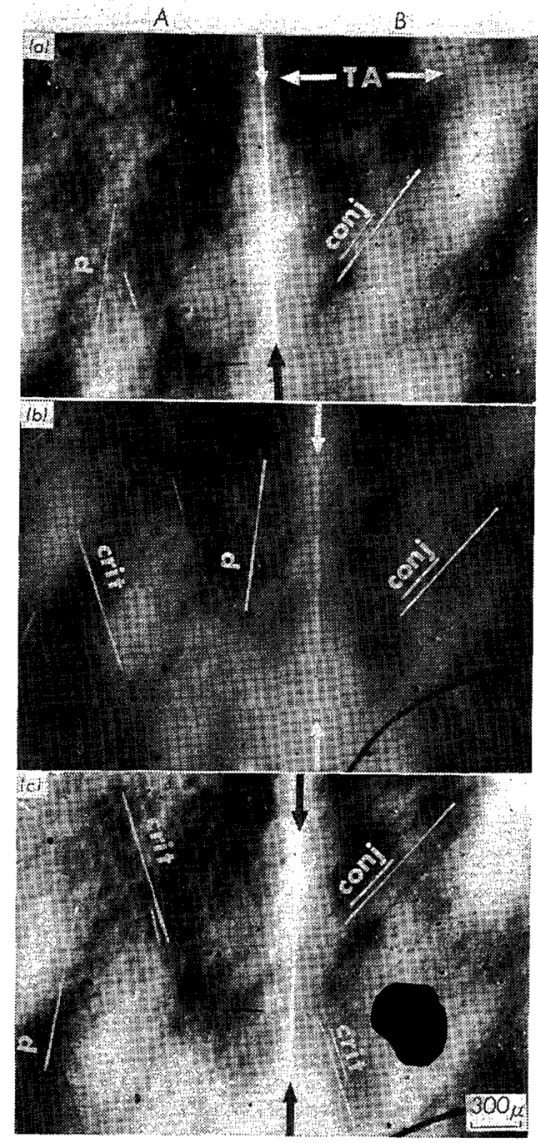

Fig. 5 Deformation of a tilt-type bicrystal with $\theta=24^{\circ}$. 

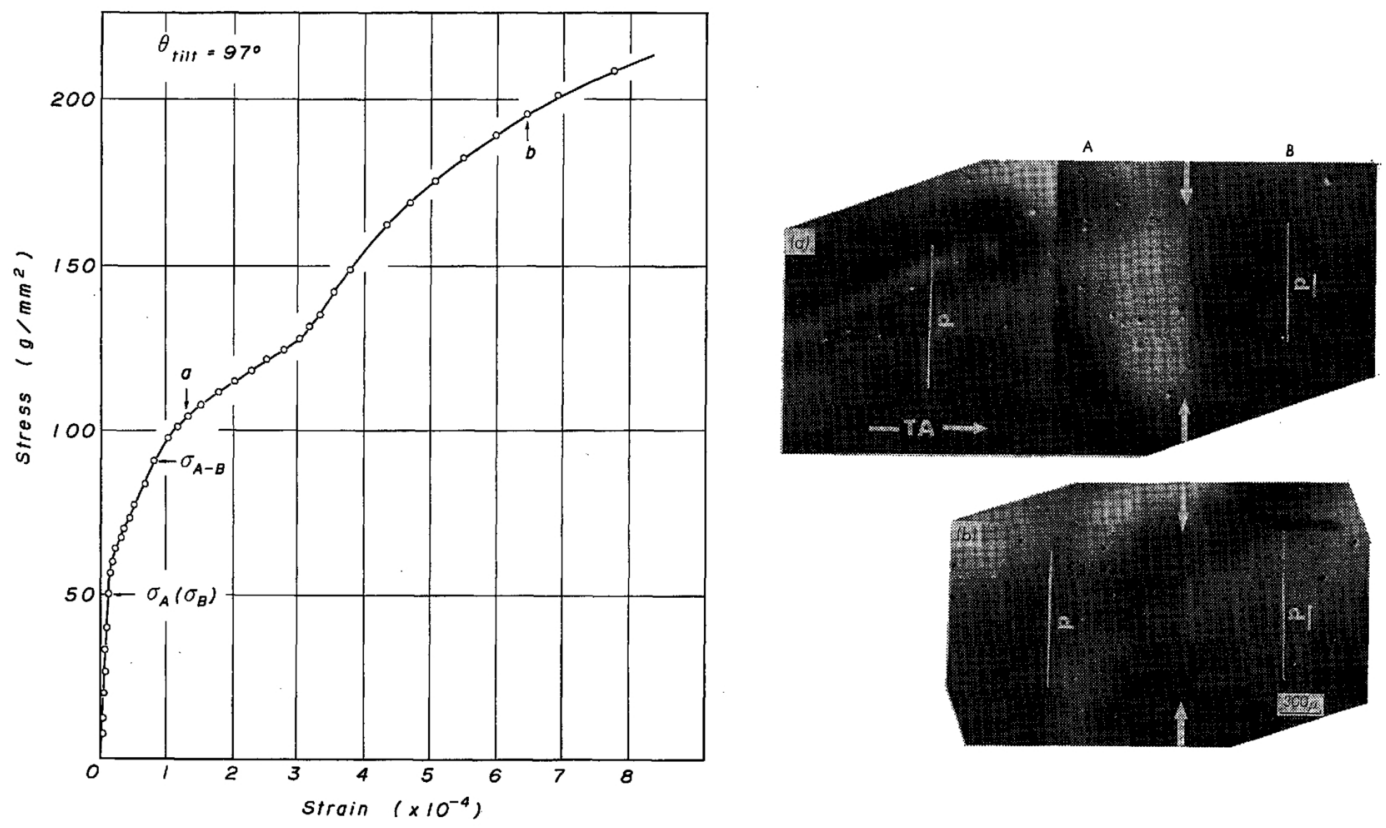

Fig. 6 Deformation of a tilt-type bicrystal with $\theta=97^{\circ}$.

tions among multiple slip systems in both crystals.

When $\theta$ increases to $24^{\circ}$, the flow stress increases rapidly even at the early stage of deformation, as seen in Fig. 5. In this case, slips $p$ and conj appear in the same amount at the early stage of deformation, as seen in micrograph (a). After that the secondary slips crit and crit become prominent in wide regions of the individual crystals, respectively. Here, it is noted that the amounts of primary slips $p$ and conj in both crystals are rather small near the boundary, and that the rapid hardening in Fig. 5 corresponds to the formation of the secondary slip bands.

Figure 6 shows a case of $\theta=97^{\circ}$. In this case, crystals $\mathbf{A}$ and $\mathbf{B}$ are in nearly symmetrical relation with each other. When the specimen is deformed, slips $p$ and $\underline{p}$ appear first. And then the flow stress rapidly increases when the strain reaches about $3 \times 10^{-4}$, though only the primary slip bands of $p$ and $\underline{p}$ are observed, as seen in micrographs (a) and (b). What is to be noted here is that slips $p$ and $\underline{p}$ are enhanced near the boundary. This fact is considered to result from the interaction between the two crystals and to be related to the rapid increase of flow stress.
From these results mentioned above, it is concluded that the affected region, where new slip systems are activated by the interaction between the two crystals, becomes narrow in a range of misfit angles of $19 \sim 24^{\circ}$ in which the continuity of slip between the main slips in the two crystals is the worst.

\section{Twist-type specimens}

Figures 7 and 8 are examples of bicrystals with a twist boundary, in which crystal $\mathbf{B}$ is twisted by 10 and $25^{\circ}$, respectively, against crystal A oriented for a Schmid factor of 0.5 . In these specimens, the continuity of slip between two crystals is widely changed compared with the change of Schmid factor of individual slips with increasing misfit angle. Even in these cases, the deformation always starts in crystal $\mathbf{A}$ by the primary slip $p$ at the same stress level, $50 \mathrm{~g} / \mathrm{mm}^{2}$, as in the case of tilt-type ones.

When the misfit angle is $10^{\circ}$, the deformation in crystal A proceeds by only slip $p$. On the other hand, slip $p$ appears in crystal $\mathbf{B}$, but the amount of slip is rather small in regions near the boundary where the secondary slip conj slightly appears even at the early stage of deformation, as seen in micrograph (a). There- 

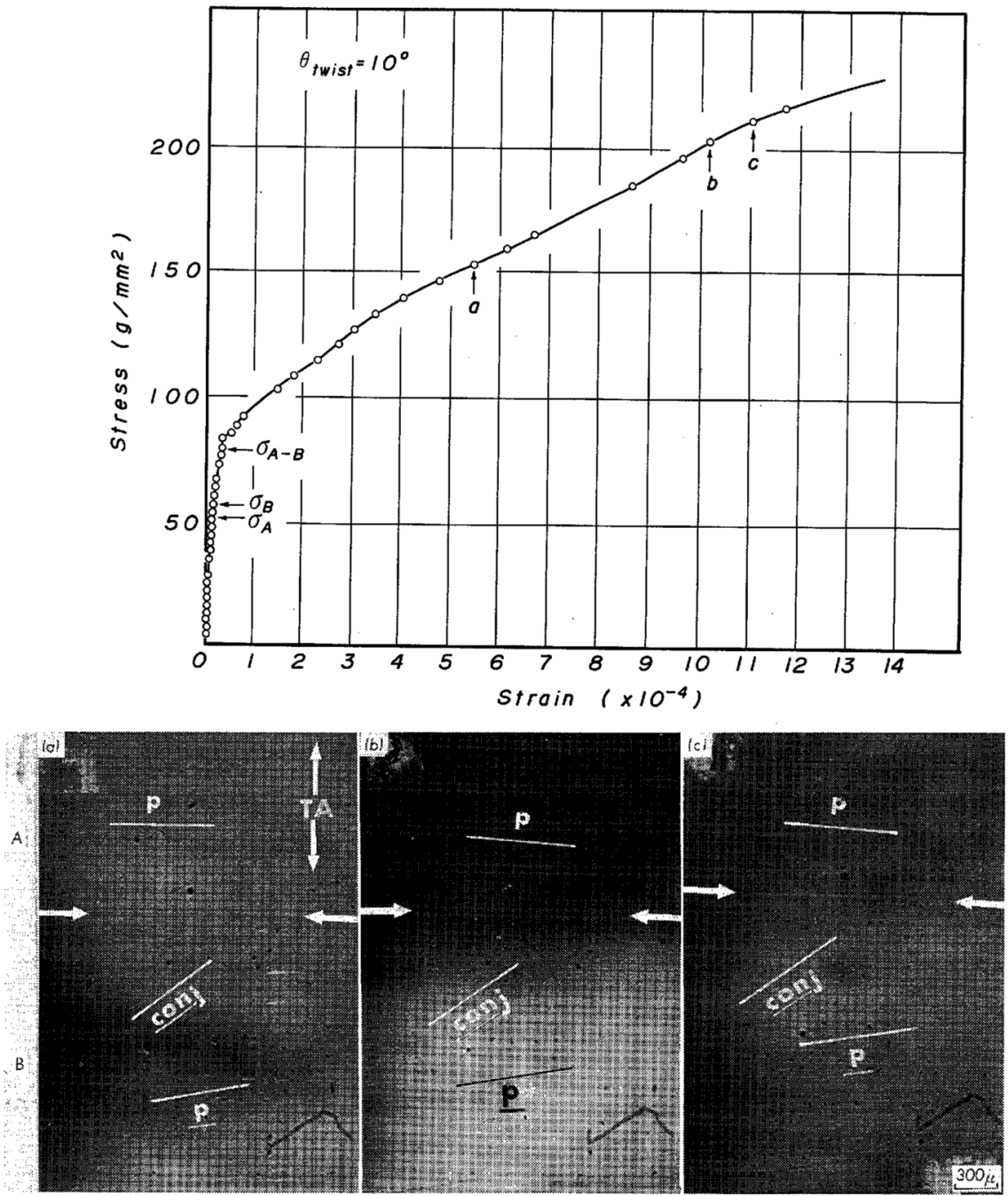

Fig. 7 Deformation of a twist-type bicrystal with $\theta=10^{\circ}$.

fore, it is considered that slip conj is associated with the interaction between two crystals and considerable work-hardening results.

When $\theta$ increases to $25^{\circ}$, slips $p$ and $\underline{p}$ appear in crystals $\mathbf{A}$ and $\mathbf{B}$ respectively at the early stage of deformation, as seen in micrograph (a). At strain $b$, slip conj appears in regions near the boundary where the amount of slip $p$ is rather small. At strain $c$, slip crit also appears in crystal $\mathbf{B}$ only in regions near the boundary where the amount of slip $\underline{p}$ is small. Slips conj and crit are very short in length so that they take an appearance of wavy slip by the combination with slips $p$ and $\underline{p}$, respectively, as seen in micrograph (c). These secondary slips conj and crit in two crystals cause the rapid work- hardening at strain $c$.

In these specimens, the affected region also becomes narrower with increasing misfit angle, at least up to $25^{\circ}$, according to the increase of discontinuity of slip between two crystals.

\section{Boundary strength}

The boundary strength is expressed by a stress at which the deformation in one grain is transmitted to the neighboring grain through the boundary. In the present experiment, the deformation always starts in crystal $\mathbf{A}$ by $\operatorname{sip} p$ at a fixed tensile stress $50 \mathrm{~g} / \mathrm{mm}^{2}\left(\sigma_{\mathrm{A}}\right)$, while slip in crystal $\mathbf{B}$ is also activated at stress $\sigma_{\mathbf{B}}$ just after the activation of slip $p$ in general. And thus the dislocations of $\operatorname{slip} p$ always tend 

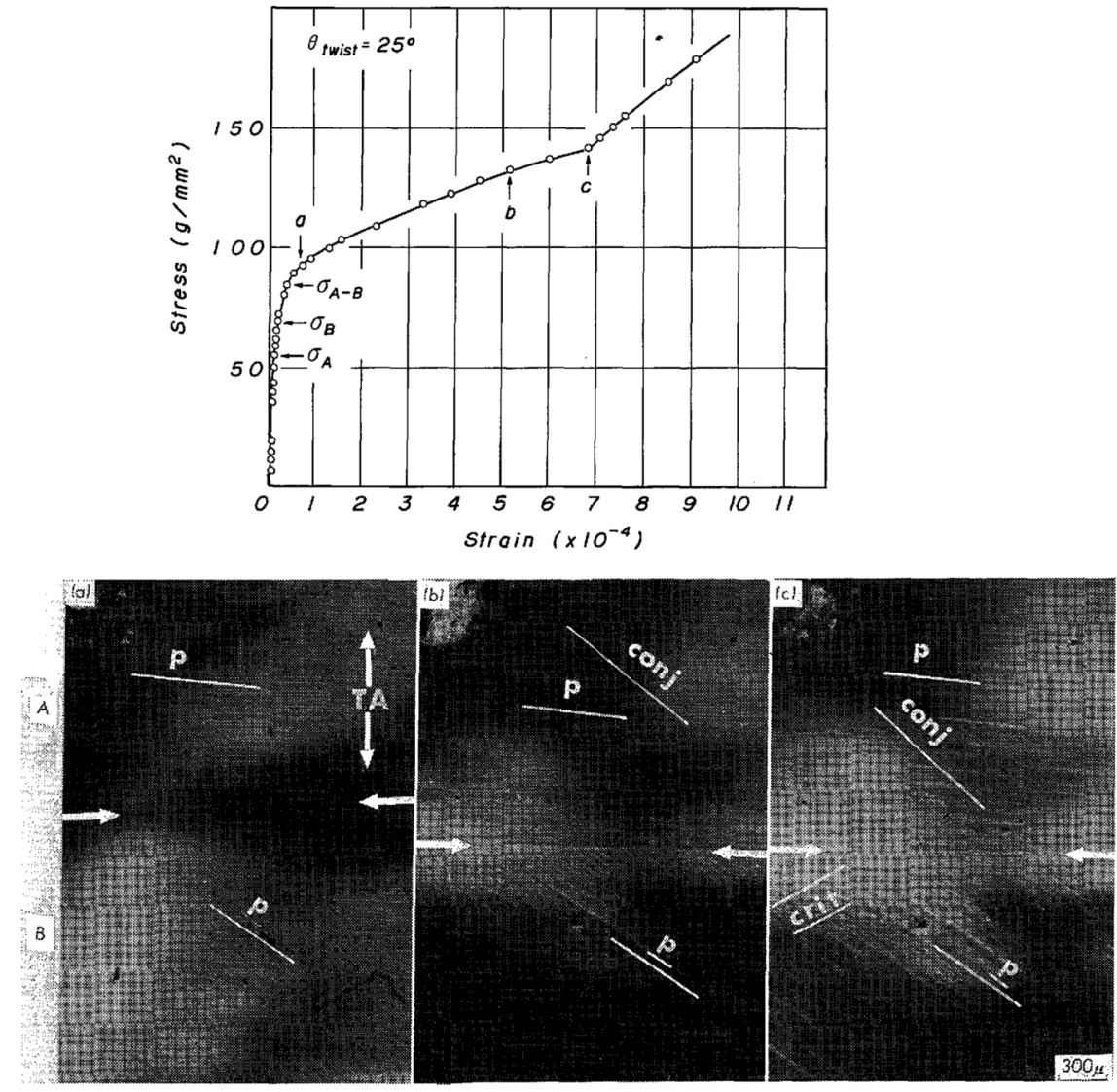

Fig. 8 Deformation of a twist-type bicrystal with $\theta=25^{\circ}$.

to move into crystal $\mathbf{B}$ through the boundary when the stress becomes higher than $\sigma_{\mathrm{A}}$. Since the slope of stress-strain curves is changed not only at $\sigma_{\mathrm{A}}$ and $\sigma_{\mathrm{B}}$ but also at stress $\sigma_{\mathrm{A}-\mathrm{B}}$, stress $\sigma_{\mathrm{A}-\mathrm{B}}$ is considered as a stress at which the deformation due to slip $p$ is transmitted considerably into crystal $\mathbf{B}$ through the boundary.

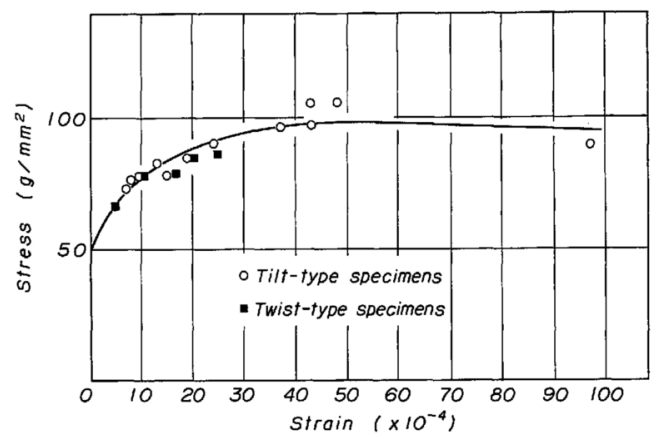

Fig. 9 Relations between the misfit angle and the strength of boundary $\left(\sigma_{\mathrm{A}-\mathrm{B}}\right)$.
Namely, stress $\sigma_{\mathrm{A}-\mathrm{B}}$ is defined as the boundary strength.

Figure 9 shows the effect of misfit angle on stress $\sigma_{\mathrm{A}-\mathrm{B}}$ in both tilt- and twist-type specimens. Here, the boundary strength in both types of specimens increases with increasing misfit angle up to about $20^{\circ}$, and then it becomes constant over the misfit angle range larger than about $20^{\circ}$.

4. Correlation between the stress-strain curves by simple tension and those by four-point bending

The present experiment have been carried out by four-point bending method. In this method, the tensile stress is changed with the depth from the surface. And thus there is an ambiguity that the deformation mode is affected by the characteristic of four-point bending when the strain increases up to $10^{-3}$ corresponding to the apparent yielding. In 

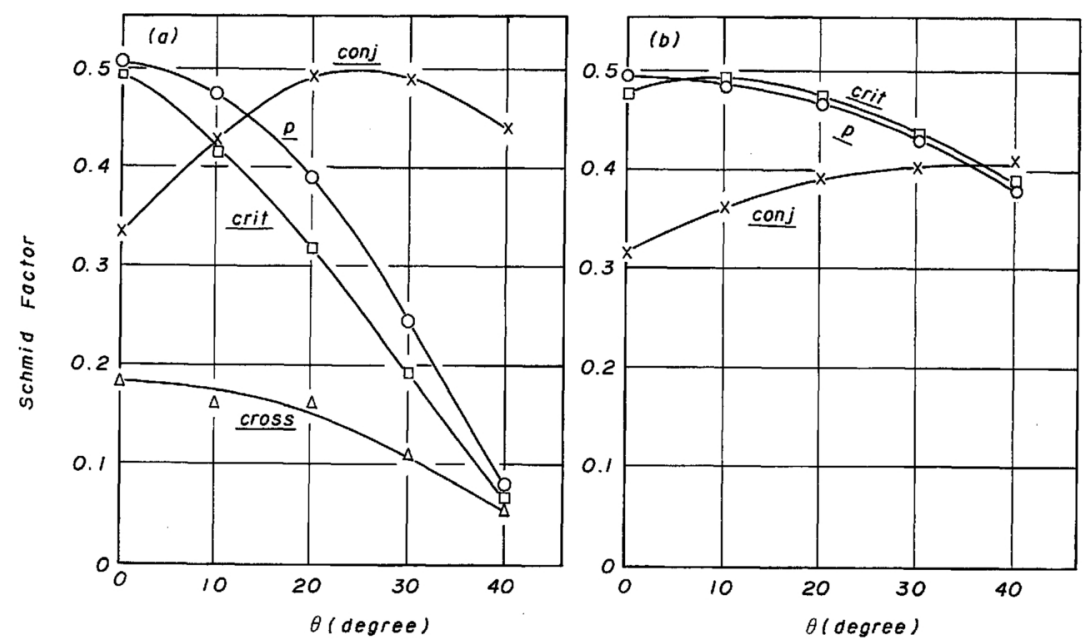

Fig. 10 Variation of the Schmid factor for each of the corresponding slip systems in crystal B against the misfit angle for (a) tilt-type and (b) twist-type bicrystals.

order to check this point, long bicrystals of $1.5 \times 40 \times 200 \mathrm{~mm}^{3}$ in size were grown by a large carbon mold with the same combination of seeds. Tensile specimens of $1.5 \times 5 \times 40$ $\mathrm{mm}^{3}$ were cut from these mother bicrystals by acid cutting, and extended along a direction perpendicular to the boundary with an Instrontype tensile machine of Shimadzu Autograph IS-5000 type. The results show that both the flow stress at about $10^{-3}$ in strain and the configuration of active slip bands in these specimens are the same as those in the case of fourpoint bending. Therefore, it is concluded that the deformation mode in the strain range less than $10^{-3}$ by four-point bending shows a good correlation with that by simple tension.

\section{Discussion}

\section{Flow stress and the active slip systems}

In the present experiment, the flow stress of bicrystals has been measured in the strain range less than $10^{-3}$. Here, it is emphasized that the plastic deformation is always initiated in crystal $\mathbf{A}$ oriented for a Schmid factor of 0.5 at a fixed stress of $50 \mathrm{~g} / \mathrm{mm}^{2}$, that is $25 \mathrm{~g} / \mathrm{mm}^{2}$ in the resolved shear stress, irrespective of the misfit angle and of the type of boundaries.

Figure 10 shows the Schmid factor for each of the corresponding slip systems in crystal B against the misfit angle $\theta$ of the boundary. In the tilt-type specimens, the Schmid factor of slip $\underline{p}$ decreases gradually with increasing misfit angle, as seen in Fig. 10(a). On the contrary, the Schmid factor of slip conj increases with increasing misfit angle up to about $25^{\circ}$ and then decreases. Therefore, it is expected in the tilt-type specimens, that the primary slip in crystal B occurs by slip $\underline{p}$ until their misfit angle increases to about $13^{\circ}$, and that the slip system changes to slip con $j$ when $\theta$ further increases. In the twist-type specimens, the Schmid factor of individual slips in crystal $\mathbf{B}$ does not change so much against the misfit angle, as seen in Fig. 10(b). Therefore, the primary slip in crystal B is expected to occur always by slip $\underline{p}$ up to about $35^{\circ}$ in the misfit angle.

These predictions show a good coincidence with the present results at the beginning of deformation.

When the strain increases considerably, another factor, i.e., the interaction between two crystals, must be taken into account. In order to check the interaction between the two crystals, the following two experiments are effectively used: One is made with bicrystals whose composite crystals are rotated with each other around the stress axis so that the Schmid factor of slip systems in both crystals does not change. Deformation of the twist-type specimens in the present experiment are close to this case. 
The other is to compare the deformation of two individual single crystals $\mathbf{A}$ and $\mathbf{B}$ with that of a bicrystal composed of these two crystals. When these three specimens $\mathbf{A}, \mathbf{B}$ and $\mathbf{A}+\mathbf{B}$ are extended separately by the four-point bending method ${ }^{(8)}$, the flow stress of bicrystal is always higher than that of anyone of two single crystals after the stress reaches $\sigma_{\mathrm{A}}$ in the strain range less than $10^{-3}$. This fact means that the flow stress of bicrystal over the stress range higher than $\sigma_{\mathrm{A}}$ is considerably affected by the interaction between crystals $\mathbf{A}$ and $\mathbf{B}$.

The interaction between two crystals is generally caused by the following two facts: (a) The localized stress associated with the active slip in the opposite crystal, which is due to the discontinuity of slip between the two crystals. (b) Additional stress caused by the difference in crystal rotation between the two crystals. In the strain range less than $10^{-3}$, the interaction is mainly related to fact (a) because of the small strain. The interaction results in the affected regions where suitable slips are activated for relieving the localized stress due to the fact (a).

Active slip planes in individual crystals of tilt- and twist-type specimens in the present experiment are summarized in Tables 2 and 4, respectively. Here it should be noted that the amount of active slip is suppressed near the boundary before new slip systems are activated

Table 2 Observed slip systems in two crystals at the early and later stages of deformation in tilt-type specimens.

\begin{tabular}{|c|c|c|c|c|c|c|}
\hline \multicolumn{2}{|c|}{$\theta$ (degree) } & 7 & 10 & 19 & 24 & 97 \\
\hline \multirow[t]{2}{*}{ crystal A } & $\mathrm{E}$ & $\stackrel{p}{p})$ & $\begin{array}{c}p^{-} \\
(0.5)\end{array}$ & $\begin{array}{c}p^{-} \\
(0.5)\end{array}$ & $\begin{array}{c}p^{-} \\
(0.5)\end{array}$ & $\begin{array}{c}p^{-} \\
(0.5)\end{array}$ \\
\hline & $\mathbf{L}$ & $p$ & $p^{-} \gg \operatorname{conj}^{+}$ & $\begin{array}{r}p^{-} \gg \operatorname{conj}^{+} \\
\left(0.3_{4}\right)\end{array}$ & $p^{-} \simeq$ crit $^{+}$ & $p^{-}$ \\
\hline \multirow[t]{2}{*}{ crystal B } & $\mathrm{E}$ & $\left(0.4_{8}\right)$ & $\frac{p^{+}}{\left(0.4_{7}\right)}$ & $\frac{c o n j^{-}}{\left(0.4_{9}\right)}$ & $\frac{c o n j}{-}$ & $\left(0.4_{7}\right)$ \\
\hline & $\mathrm{L}$ & $\underline{p}^{+}$ & $\underline{p}^{+}>\frac{c o n j^{-}}{\left(0.4_{3}\right)}$ & $\underline{\text { conj }}^{-} \gg \underbrace{\text { crit }^{+}}_{\left.(\overline{0.4})_{3}\right)} \simeq\left(\underline{0.3}_{7}^{+}\right)$ & 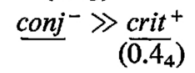 & $\underline{p}^{+}$ \\
\hline \multicolumn{2}{|c|}{$\sigma_{\mathrm{A}-\mathrm{B}}\left(\mathrm{g} / \mathrm{mm}^{2}\right)$} & 75 & 80 & 85 & 95 & 85 \\
\hline
\end{tabular}

Index (-) of slip systems indicates that the amount of slip decreases near the boundary, and index $(+)$ the contrary. Relative amount of individual slips is expressed by each inequality, and the Schmid factor of slip is shown in each parenthesis.

Notations $\mathrm{E}$ and $\mathrm{L}$ show the early stage $\left(1 \sim 2 \times 10^{-4}\right.$ strain $)$ and the later stage $\left(5 \sim 10 \times 10^{-4}\right.$ strain $)$ of deformation, respectively.

Table 3 Estimated modes of slip-transmission between two crystals in tilt-type specimens.

\begin{tabular}{|c|c|c|c|c|c|}
\hline$\theta$ (degree) & 0 & 10 & 20 & 30 & 40 \\
\hline \multirow{3}{*}{$\begin{array}{l}\text { Estimated } \\
\text { slip-transmission } \\
\text { between two } \\
\text { crystals } \mathbf{A} \text { and } \mathbf{B}\end{array}$} & $\begin{array}{c}p \rightarrow \underline{p} \\
(0.5)(0.5) \\
(M=1)\end{array}$ & $\begin{array}{c}p \rightarrow \underline{p} \\
(0.5)\left(0.4_{7}\right) \\
\left(M=0.9_{4}\right)\end{array}$ & $\begin{array}{l}\frac{\text { conj } \rightarrow \text { conj }}{\left(0.4_{9}\right)\left(0.3_{3}\right)} \\
\left(M=0.7_{9}\right)\end{array}$ & $\begin{array}{l}\text { conj } \rightarrow \text { crit } \\
\left(0.4_{9}\right)\left(0.4_{9}\right) \\
\left(M=0.9_{5}\right)\end{array}$ & 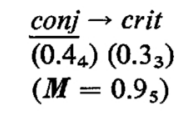 \\
\hline & & $\begin{array}{c}\frac{\text { conj } \rightarrow \text { conj }}{\left(0.4_{3}\right)\left(0.3_{3}\right)} \\
\left(M=0.9_{5}\right)\end{array}$ & $\begin{array}{c}\text { conj } \rightarrow \text { crit } \\
\left(0.4_{9}\right)\left(0.4_{4}\right) \\
\left(M=0.8_{7}\right)\end{array}$ & $\begin{array}{l}\text { crit } \rightarrow \text { crit } \\
\left(0.4_{9}\right)\left(0.4_{4}\right) \\
\left(M=0.7_{1}\right)\end{array}$ & $\begin{array}{l}\text { crit } \rightarrow \frac{\text { crit }}{\left(0.4_{3}\right)} \\
\left(0.3_{3}\right) \cdot\left(0.6_{8}\right)\end{array}$ \\
\hline & & & $\begin{array}{c}p \rightarrow \underline{p} \\
(0.5)\left(0.3_{4}\right) \\
\left(M=0.7_{6}\right)\end{array}$ & 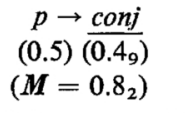 & $\begin{array}{c}p \rightarrow \frac{\text { conj }}{\left(0.4_{4}\right)} \\
(0.5)\left(M=0.7_{4}\right)\end{array}$ \\
\hline
\end{tabular}

Notation $M$ shows a calculated value in the parenthesis in eq. (1) when the right-hand side slip system is activated by the left-hand side slip system. The Schmid factor of each individual slip system is also indicated in each parenthesis just below each notation of the slip system. 
Table 4 Observed slip systems in two crystals at the early and later stages of deformation in twist-type specimens.

\begin{tabular}{cccc}
\hline \multicolumn{2}{r}{$\alpha$ (degree) } & 10 & 25 \\
\hline crystal A & $\mathrm{E}$ & $p^{-}$ & $p^{-}$ \\
& $\mathrm{L}$ & $p^{-}$ & $p^{-} \gg c o n j^{+}$ \\
\hline crystal B & $\mathrm{E}$ & $\underline{p}^{-} \gg \frac{c o n j^{+}}{\underline{c o n j}^{+}}$ & $\underline{p}^{-} \underline{p}^{-}$ \\
& $\mathrm{L}$ & $\underline{c r i t}^{+}$ \\
\hline \hline
\end{tabular}

in the affected region.

Now it is assumed that new slip systems are activated by the additional shear stress associated with a slip in the opposite crystal, as calculated by Livingston and Chalmers ${ }^{(10)}$. Namely, the additional shear stress $\tau_{i}$ on slip system $i$ in crystal $\mathbf{B}$, which has $e_{i}$ as its slip plane normal and $g_{i}$ as its slip direction, can be expressed by

$$
\tau_{i}=\tau_{0}\left[\left(e_{0} \cdot e_{i}\right)\left(g_{0} \cdot g_{i}\right)+\left(e_{0} \cdot g_{i}\right)\left(e_{i} \cdot g_{0}\right)\right],
$$

where $\tau_{0}$ is the shear stress through the boundary which is associated with a slip system $o$ in crystal $\mathbf{A}$ which has a slip plane normal $e_{0}$ and a slip direction $g_{0}$.

Tables 3 and 5 show the calculated values of a parenthesized factor, which is named the transmission factor $(\boldsymbol{M})$ here, in eq. (1) over the misfit angle range of $0 \sim 40^{\circ}$ for tilt-type specimens and of $0 \sim 25^{\circ}$ for twist-type specimens, respectively.

By comparing Tables 2 with 3 and 4 with 5 respectively, it is recognized that the active slip system observed in the affected region shows a good coincidence with those estimated from eq. (1).

For example, in a case of tilt-type specimen with $\theta=10^{\circ}$, a slip system with a Schmid factor of 0.5 is activated in crystal $\mathbf{A}$ first, and then slip $\underline{p}$ is activated in crystal B. Since $\boldsymbol{M}$ of $p \rightarrow \underline{p}$ is $0.9_{4}$, slip $\underline{p}$ is promoted by slip $p$ near the boundary and slip $p$ is suppressed near the boundary. After that slip $\underline{p}$ expands all over crystal $\mathbf{B}$ because of its large Schmid factor, and then slip conj is also activated in crystal $\mathbf{B}$ in sequence of the Schmid factor. This slip conj activates slip conj in crystal $\mathbf{A}$ through the boundary when the stress increases further, because $\boldsymbol{M}$ of conj $\rightarrow$ conj is so large as $0.9_{5}$. Since the Schmid factor of slip conj is not so large, slip conj is limited to appear in regions near the boundary. Other cases in Tables 2 and 4 can also be correlated with Tables 3 and 5 respectively in the same way.

As mentioned above, the width of the affected region is a function of $M$ and the Schmid factor of individual slips. The value of $\boldsymbol{M}$ decreases with increasing discontinuity of slip between individual slips $o$ and $i$ in the two crystals, and shear stress $\tau_{i}$ acts on the active slip planes of slip $i$ in addition to the resolved shear stress of the applied stress. Therefore, the width of the affected region decreases with decrease not only in $\tau_{i}$ but also in Schmid factor of activated slip $i$. Furthermore, so-called unpredicted slips, whose Schmid factor is smaller than about 0.3, are also activated near the boundary when the value of $\tau_{i}$ is fairly large even in the strain range smaller than $10^{-3}$.

Table 5 Estimated modes of slip-transmission between two crystals in twist-type specimens.

\begin{tabular}{|c|c|c|}
\hline$\theta$ (degree) & 10 & 25 \\
\hline \multirow{3}{*}{$\begin{array}{l}\text { Estimated } \\
\text { slip-transmission } \\
\text { between two crystals } \\
\text { A and B }\end{array}$} & $\begin{array}{c}p_{\mathrm{I}} \rightarrow \underline{p}_{\mathrm{I}} \\
(0.5)\left(0.4_{9}\right) \\
\left(\boldsymbol{M}=0.9_{8}\right)\end{array}$ & $\begin{array}{c}p_{\mathrm{I}} \rightarrow \underline{p}_{\mathrm{I}} \\
(0.5)\left(0.4_{5}\right) \\
\left(\boldsymbol{M}=0.9_{1}\right)\end{array}$ \\
\hline & $\begin{array}{c}p_{\mathrm{I}} \rightarrow \frac{c o n j_{\mathrm{III}}}{\left(0.3_{6}\right)} \\
(0.5) \\
\left(\boldsymbol{M}=0.7_{4}\right)\end{array}$ & $\begin{array}{c}\underline{p}_{\mathrm{I}} \rightarrow \operatorname{conj}_{\mathrm{III}} \\
\left(0.4_{5}\right)\left(0.2_{6}\right) \\
\left(\boldsymbol{M}=0.8_{5}\right)\end{array}$ \\
\hline & & $\begin{array}{l}\underset{p_{\mathrm{I}} \rightarrow}{\rightarrow} \frac{\text { crit }_{\mathrm{I}}}{(0.5)}\left(0.4_{5}\right) \\
\left(M=0.7_{9}\right)\end{array}$ \\
\hline
\end{tabular}

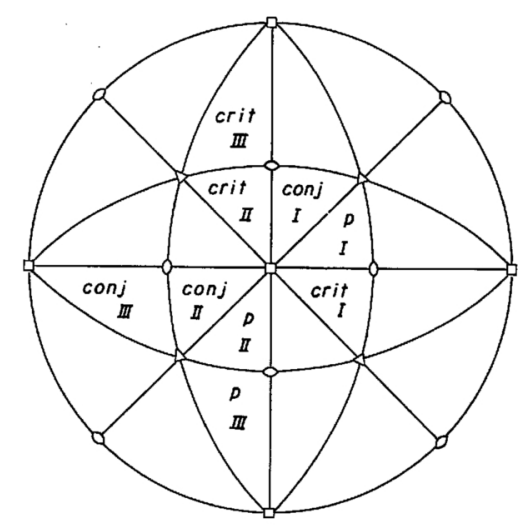




\section{Apparent yield stress and geometrical relation between two crystals}

It was shown in the present experiment that the rapid work hardening occurs in regions near the boundary by the interaction between two crystals even in the strain range less than $10^{-3}$. The interaction is closely related to the misfit angle of the boundary, and the flow stress at a strain of $10^{-3}$, which roughly corresponds to the apparent yield stress, increases with increasing misfit angle at least up to $24^{\circ}$ in the case of tilt-type specimens.

The apparent yield stress, however, sometimes deviates from this rule. Figure 11 shows an example of such exceptions in the case of tilt-type specimens. Here, the stress-strain curves shown by a chain and a solid line correspond to the specimens with $\theta=+7^{\circ}$, which is the same as that in Fig. 3, and $-8^{\circ}$, respectively. In Fig. 11, the flow stress of a specimen with $\theta=-8^{\circ}$ is always lower than that of $\theta=+7^{\circ}$ over the strain range less than $10^{-3}$. In this case, it is noted that the Schmid factor of $\operatorname{sip} p$ in crystal $\mathbf{A}$ of a specimen with $\theta=-8^{\circ}$ is slightly smaller than 0.5 , and that crystal $\mathbf{B}$ is rotated against crystal $\mathbf{A}$ in the reverse direction to the case of $\theta=+7^{\circ}$. Practically the Schmid factors of slips $p$ and $\underline{p}$ are $0.4_{9}$ and $0.4_{8}$ respectively, and the transmission factor of $\operatorname{slip} p \rightarrow \underline{p}$ becomes $0.9_{8}$. And thus, the transmission of slip $p \rightarrow \underline{p}$ easily occurs in this case compared with the case of $\theta=7^{\circ}$. Therefore, the work hardening rate in a specimen with $\theta=-8^{\circ}$ becomes smaller compared with that in a case of $\theta=+7^{\circ}$, because the accumulated stress on the boundary is easily relieved by slip $\underline{p}$.

Namely, the difference in flow stress between two specimens in Fig. 11 is not related to a special reason such as the generation of dislocation from the boundary, but attributed to the

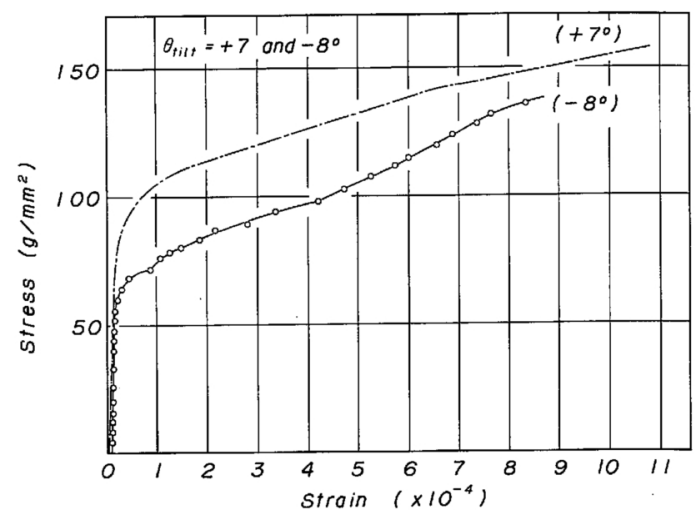

Fig. 11 Flow stress and the sign of misfit angle. Two crystals of a specimen with $\theta=-8^{\circ}$ mutually rotate reversely against those with $\theta=+7^{\circ}$. The former shows the lower flow stress than that of the latter.

easiness of the transmission of slips between the two crystals.

In conclusion, the flow stress of bicrystals in the strain range less than $10^{-3}$ depends not only on the misfit angle of the boundary, but also on the combination of the Schmid factor and the transmission factor of individual slips in the two crystals.

\section{REFERENCES}

(1) E. O. Hall: Proc. Phys. Soc. (London), 64 (1951), 747.

(2) N. J. Petch: J. Iron Steel Inst., 174 (1953), 25.

(3) B. Chalmers: Proc. Roy. Soc., A162 (1937), 120.

(4) B. Chalmers: Canad. J. Phys., 31 (1953), 132.

(5) J. Gilman: Acta Met., 1 (1953), 426.

(6) S. Karashima: Memoirs Inst. Sci. Ind. Res., Osaka University, 10 (1952), 78.

(7) R. E. Hook and J. P. Hirth: Acta Met., 15 (1967), 535; 1099.

(8) H. Fujita, K. Toyoda and J. Abu: Read at the annual meeting of the Japan Inst. Metals, April (1972).

(9) H. Mori and H. Fujita: Materials Science and Engineering, 13 (1974), 41.

(10) J. D. Livingston and B. Chalmers: Acta Met., 5 (1957), 322. 